\title{
Single qubit measurements with an asymmetric single-electron transistor
}

\author{
S.A. Gurvitz ${ }^{1,2}$ and G.P. Berman ${ }^{2}$ \\ ${ }^{1}$ Department of Particle Physics, Weizmann Institute of Science, Rehovot 76100, Israel \\ ${ }^{2}$ Theoretical Division, Los Alamos National Laboratory, Los Alamos, NM 87545, USA
}

\begin{abstract}
We investigate qubit measurements using a single electron transistor (SET). Applying the Schrödinger equation to the entire system we find that an asymmetric SET is considerably more efficient than a symmetric SET. Yet, its efficiency does not reach that of an ideal detector even in the large asymmetry limit. We also compare the SET detector with a point-contact detector. This comparison allows us to illuminate the relation between information gain in the measurement process and the decoherence generated by these measurement devices.
\end{abstract}

PACS: 73.50.-h, 73.23.-b, 03.65.Yz.

An obvious candidate for read-out of the two-state system (qubit) is the single electron transistor (SET) [1,2]. In many respects it is better than the point-contact (PC) detector [1], which has already been used for quantum measurements [3]. It has been shown, however, that the symmetric SET has a rather low sensitivity in its normal working regime $[4,5]$. Therefore it becomes very important to investigate how to improve the effectiveness of the SET by a proper selection of its parameters.

In this Letter we examine qubit measurements using the SET by applying the Schrödinger equation to the entire system of the qubit and detector. In this case, we can unambiguously determine the backaction of the charge fluctuations in the SET on the qubit and the sensitivity of the measurement as a function of the detector parameters. We find that by varying the tunneling barriers of the SET, one can force the latter to operate in a regime where the "active measurement" time is very short. Then the SET behaves as a linear quantum detector even if it is strongly coupled to the measured system. We also demonstrate that in this regime the effectiveness of the SET considerably increases, although it does not reach the ultimate value corresponding to an ideal detector. By varying the set-up parameters of the SET one can investigate decoherence generated by this detector in the operation mode in which the signal decreases to zero. This illuminates the relationship between decoherence and distinguishability in different types of measurements.

Consider an electrostatic qubit, represented by an electron in a coupled dot. The qubit is placed in close proximity to the SET, Fig. 1. The latter is shown as a potential well, coupled to two reservoirs at different chemical potentials, $\mu_{L, R}$. We assume that the bias voltage $V=\mu_{L}-\mu_{R}$ is smaller than the energy spacing of the single particle states of the well. This condition is usually realized in semiconductor quantum dots [6]. Then transport takes place through an individual single particle level, $E_{0}$, in contrast to a metallic SET, in which many conducting levels contribute to transport [7]. One finds that if the electron occupies the upper dot (Figs. 1a,1b) the current flows through the level $E_{0}$ of the SET. However, if the electron occupies the lower dot (Figs. 1c,1d), the SET is blocked due to the electronelectron repulsion $U$. Here we assume that $U$ is large enough so that $E_{0}+U-\mu_{L} \gg \Gamma_{L, R}$, where $\Gamma_{L, R}$ are the partial widths of the level $E_{0}$ due to coupling with the reservoirs. Thus the probability of finding the qubit in the state $E_{1}$ (Fig. 1a,1b) would be given by the ratio, $I(t) / I_{0}$, where $I(t)$ is the (ensemble) average detector current and $I_{0}=e \Gamma_{L} \Gamma_{R} /\left(\Gamma_{L}+\Gamma_{R}\right)$ is the detector current in the absence of a qubit.

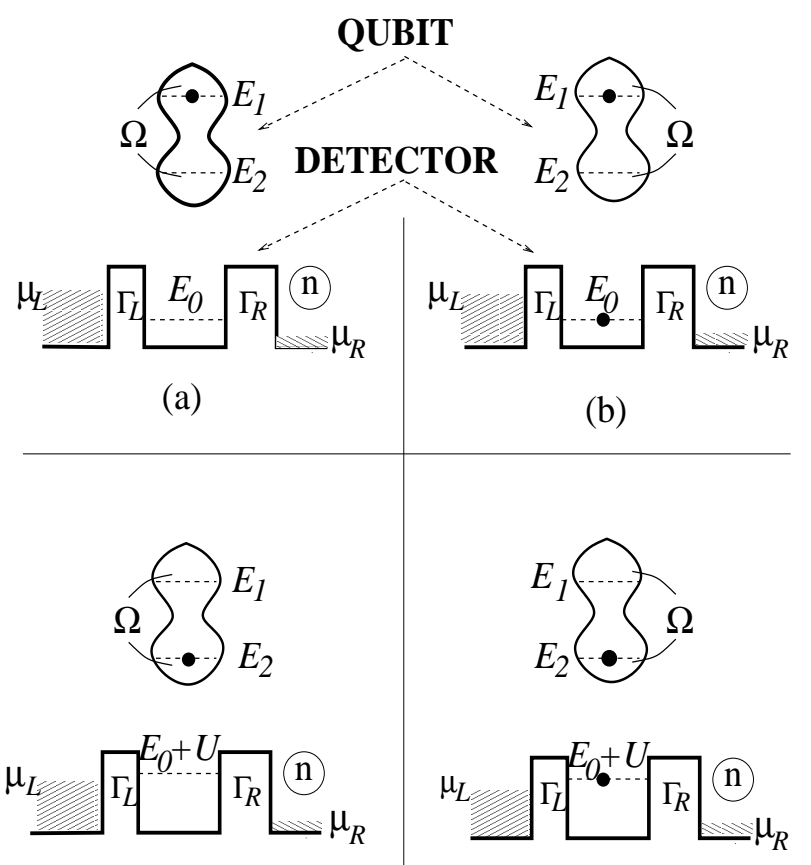

(c)

(d)

Fig. 1: Possible electron configurations of the measured qubit and the detector. Here $n$ denotes the number of electrons which have arrived at the right reservoir by time $t$.

The ideal linear measurement, however, cannot be fully realized since the electric current would flow through the SET even if the electron of the qubit occupies the lower dot (Fig. 1d) [8]. In addition the state (1b) prevents qubit oscillations due to the electron-electron repulsion 
$U$. As a result the average detector current could not follow the qubit behavior.

Thus, in order to increase the effectiveness of the detector, we must diminish the role of the states $1 \mathrm{~b}$ and $1 \mathrm{~d}$ in the measurement dynamics. This can be realized by using an asymmetric SET with $\Gamma_{R} \gg \Gamma_{L}$, since the probability of finding an electron inside the quantum well of the SET is $\Gamma_{L} /\left(\Gamma_{L}+\Gamma_{R}\right)$. Then the SET detector spends most of the time in the states $1 \mathrm{a}$ and $1 \mathrm{c}$, where no interaction with the qubit takes the place. This is similar to the "interaction-free" measurement, discussed in the literature [9], which distorts the measured quantum system in a minimal way. Yet, by enhancing the asymmetry of the detector one slows down the measurement rate of the detector, as well as the fluctuations of the detector current. Therefore in order to substantiate our qualitative arguments, one needs to evaluate explicitly the detector efficiency, which involves the ratio of these two quantities.

Let as consider the entire system as described by the following tunneling Hamiltonian: $H=H_{q}+H_{\text {set }}+H_{\text {int }}$, where

$$
\begin{aligned}
& H_{q}=E_{1} a_{1}^{\dagger} a_{1}+E_{2} a_{2}^{\dagger} a_{2}+\Omega\left(a_{1}^{\dagger} a_{2}+a_{2}^{\dagger} a_{1}\right) \quad \text { and } \\
& H_{\text {set }}=H_{0}+E_{0} c_{0}^{\dagger} c_{0}+\sum_{\lambda}\left(\Omega_{\lambda}^{L} c_{0}^{\dagger} c_{\lambda}^{L}+\Omega_{\lambda}^{R} c_{0}^{\dagger} c_{\lambda}^{R}+H . c .\right)
\end{aligned}
$$

are the qubit and the SET Hamiltonians, respectively. $H_{0}=\sum_{\lambda}\left[E_{\lambda}^{L}\left(c_{\lambda}^{L}\right)^{\dagger} c_{\lambda}^{L}+E_{\lambda}^{R}\left(c_{\lambda}^{R}\right)^{\dagger} c_{\lambda}^{R}\right]$ describes the reservoirs. Here $a^{\dagger}(a)$ is the creation (annihilation) operator for the electron in the qubit and $c^{\dagger}(c)$ is the same operator for the SET; $\Omega$ is the coupling between the states $E_{1,2}$ of the qubit, and $\Omega_{\lambda}^{L, R}$ are the couplings between the level $E_{0}$ and the level $E_{\lambda}^{L, R}$ in the left (right) reservoir. The corresponding tunneling rates $\Gamma_{L, R}$ are therefore $\Gamma_{L, R}=2 \pi \rho_{L, R} \Omega_{L, R}^{2}$, where $\rho_{L, R}$ are the density of states in the reservoirs. In addition, we assumed a weak energy dependence of the couplings, $\Omega_{\lambda}^{L, R} \simeq \Omega_{L, R}$. The interaction between the qubit and the SET is described by $H_{\text {int }}=U a_{2}^{\dagger} a_{2} c_{0}^{\dagger} c_{0}$

It was demonstrated in Ref. [10] that in the limit of large $V$ and $U$ (Fig. 1), the Schrödinger equation for the entire system, $i \partial_{t}|\Psi(t)\rangle=H|\Psi(t)\rangle$, can be reduced to a Bloch-type rate equation describing the reduced density-matrix of the entire system, $\sigma_{j j^{\prime}}^{n}(t)$, where $j, j^{\prime}=\{a, b, c, d\}$, and the index $n$ denotes the number of electrons which have arrived at the right reservoir by the time $t$ (Fig. 1). This reduction of the Schrödinger equation takes place after partial tracing over the reservoir states, and it becomes exact in the large bias limit without any explicit use of any Markov-type or weak coupling approximations. The diagonal terms of this density matrix $\sigma_{j j}^{n}(t)$ are the probabilities of finding the system in one of the states shown in Fig. 1. The off-diagonal matrix elements ("coherencies"), describe the linear superposition of these states. As a result we arrive at the following Bloch-type equations describing the entire system $[10,11]$

$$
\begin{aligned}
\dot{\sigma}_{a a}^{n} & =-\Gamma_{L} \sigma_{a a}^{n}+\Gamma_{R} \sigma_{b b}^{n-1}+i \Omega\left(\sigma_{a c}^{n}-\sigma_{c a}^{n}\right), \\
\dot{\sigma}_{b b}^{n} & =-\Gamma_{R} \sigma_{b b}^{n}+\Gamma_{L} \sigma_{a a}^{n}+i \Omega\left(\sigma_{b d}^{n}-\sigma_{d b}^{n}\right), \\
\dot{\sigma}_{c c}^{n} & =\Gamma_{L} \sigma_{d d}^{n}+\Gamma_{R} \sigma_{d d}^{n-1}+i \Omega\left(\sigma_{c a}^{n}-\sigma_{a c}^{n}\right), \\
\dot{\sigma}_{d d}^{n} & =-\left(\Gamma_{L}+\Gamma_{R}\right) \sigma_{d d}^{n}+i \Omega\left(\sigma_{d b}^{n}-\sigma_{b d}^{n}\right), \\
\dot{\sigma}_{a c}^{n} & =i \epsilon \sigma_{a c}^{n}+i \Omega\left(\sigma_{a a}^{n}-\sigma_{c c}^{n}\right)-\frac{\Gamma_{L}}{2} \sigma_{a c}^{n}+\Gamma_{R} \sigma_{b d}^{n-1}, \\
\dot{\sigma}_{b d}^{n} & =i \epsilon_{1} \sigma_{b d}^{n}+i \Omega\left(\sigma_{b b}^{n}-\sigma_{d d}^{n}\right)-\left(\Gamma_{R}+\frac{\Gamma_{L}}{2}\right) \sigma_{b d}^{n},
\end{aligned}
$$

where $\epsilon=E_{2}-E_{1}$ and $\epsilon_{1}=E_{2}-E_{1}+U$.

Solving Eqs. (2) we can determine the time evolution of the qubit and the detector during the measurement process. Indeed, the qubit behavior is described by the corresponding (reduced) density matrix $\sigma_{Q B}(t) \equiv\left\{\sigma_{\alpha \alpha^{\prime}}(t)\right\}$ with $\alpha, \alpha^{\prime}=\{1,2\}$, obtained by tracing the detector variables $(n): \sigma_{11}=\sum_{n}\left(\sigma_{a a}^{n}+\sigma_{b b}^{n}\right), \sigma_{12}=\sum_{n}\left(\sigma_{a c}^{n}+\sigma_{b d}^{n}\right)$. Here $\sigma_{11}$ and $\sigma_{22}=1-\sigma_{11}$ are the probabilities of finding the qubit in the states $E_{1,2}$ respectively, and $\sigma_{12}$ describes the linear superposition of these states.

The detector behavior, on the other hand, is obtained from $\sigma_{j j^{\prime}}^{n}(t)$ by tracing over the qubit variables. For instance, the probability of finding $n$ electrons in the collector at time $t$ is $P_{n}(t)=\sum_{j} \sigma_{j j}^{n}(t)$. This quantity allows us to determine the average detector current,

$$
\left.I(t)=e \sum_{n} n \dot{P}_{n}(t)=e \Gamma_{R}\left[\sigma_{b b}(t)+\sigma_{d d}(t)\right]\right]
$$

where $\sigma_{j j^{\prime}}=\sum_{n} \sigma_{j j^{\prime}}^{n}$, and the shot-noise power spectrum, $S(\omega)$, is given by the McDonald formula [12],

$$
S(\omega)=2 e^{2} \omega \int_{0}^{\infty} d t \sin (\omega t) \sum_{n} n^{2} \dot{P}_{n}(t),
$$

One finds from Eqs. (2), (4) that

$$
S(\omega)=2 e^{2} \omega \Gamma_{R} \operatorname{Im}\left[Z_{b b}(\omega)+Z_{d d}(\omega)\right],
$$

where $Z_{j j^{\prime}}(\omega)=\int_{0}^{\infty} \sum_{n}(2 n+1) \sigma_{j j^{\prime}}^{n}(t) \exp (i \omega t) d t$. These quantities are obtained directly from Eqs. (2) which are reduced to a system of linear algebraic equations after the corresponding integration over $t$.

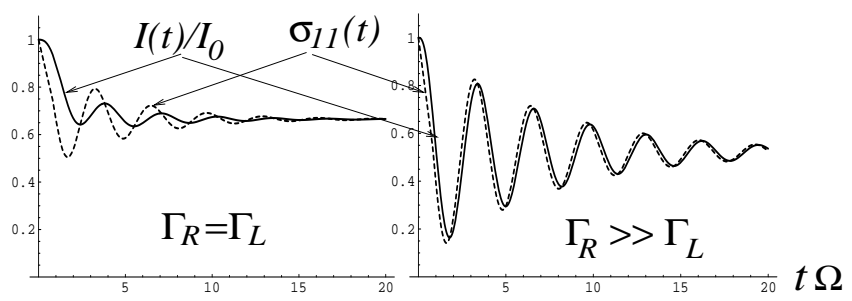

(a)

(b)

Fig. 2: The probability of finding the qubit electron in the upper dot, $\sigma_{11}(t)$ (dashed line) in comparison with $I(t) / I_{0}$ (solid line) as a function of the time $t$ for $U=20 \Omega$ and (a) $\Gamma_{L}=\Gamma_{R}=0.5 \Omega$ and (b) $\Gamma_{L}=0.5 \Omega, \Gamma_{R}=5 \Omega$. 
Consider first the average detector current, $I(t)$, Eq. (3). We can investigate the linearity of the measurement [13] by comparing the ratio $I(t) / I_{0}$ with $\sigma_{11}(t)$. These quantities are shown in Fig. 2 for a symmetric SET (a) and an asymmetric SET (b) as a function of time for $\epsilon=0$. The initial condition corresponds to the qubit electron in the upper dot and the detector current $I=I_{0}$. This implies that $\sigma_{a a}(0)=\Gamma_{R} /\left(\Gamma_{L}+\Gamma_{R}\right)$, $\sigma_{b b}(0)=\Gamma_{L} /\left(\Gamma_{L}+\Gamma_{R}\right)$, while all other $\sigma_{j j^{\prime}}(0)=0$.

One finds from Fig. 2a that for a symmetric SET the detector current does not follow the qubit oscillations and the qubit behavior is strongly distorted. As we suggested above this is caused by the configurations (b) and (d) in Fig. 1. The result of our calculations for an asymmetric SET, shown in Fig. 2b, confirms these qualitative arguments. Indeed the weight of these configurations is strongly reduced in this case. (In fact, in the case of $U \gg \Omega, \Gamma$, considered in Fig. 2, the contribution (d) remains very small even for the symmetric SET). As a result, the Rabi oscillations of the qubit are very well reproduced by the ratio $I(t) / I_{0}$ and the qubit is distorted much less than in the case of the symmetric SET.

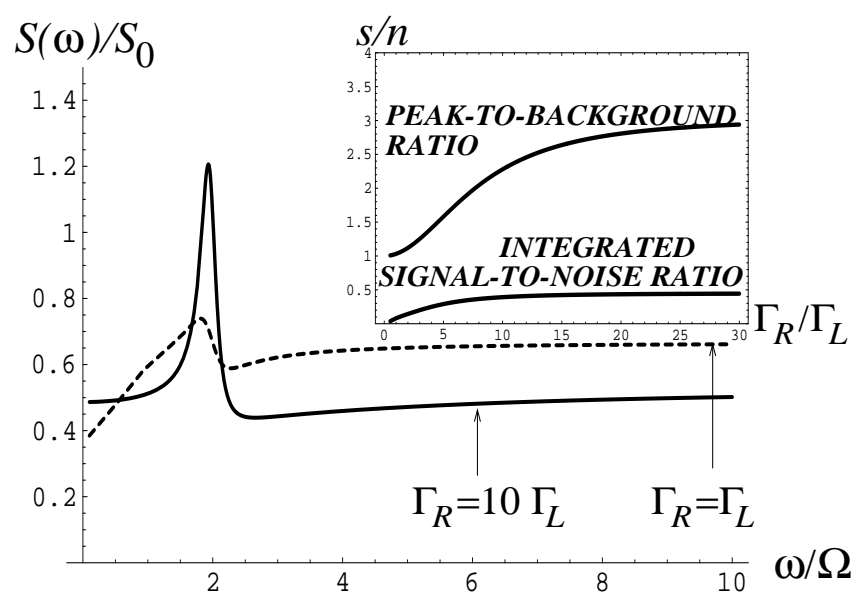

Fig. 3: Shot-noise power spectrum for the symmetric (dashed line) and the asymmetric (solid line) SET. The parameters are the same as in Fig. 2. The peak tobackground ratio and the integrated signal-to-noise ratio are shown in the inset as a function of $\Gamma_{R} / \Gamma_{L}$.

The result shown in Fig. 2 demonstrates the linearity of measurement achieved by the asymmetric SET. Yet, the ratio $I(t) / I_{0}$ does not yield the effectiveness of measurement. The latter is reflected in the detector noise spectrum (Fano factor), $S(\omega) / S_{0}$, where $S(\omega)$ is given by Eqs. (4), (5), and $S_{0}=2 e I_{0}$. The results for symmetric and asymmetric SET are shown in Fig. 3. One finds that the peak in the spectrum corresponding to the Rabi frequency of the qubit is much more pronounced for the asymmetric SET. It was argued in $[4,14]$ that a measure of a measurement efficiency is the peak-to-background ratio of the detector current power spectrum, with a per- fectly efficient detector yielding a ratio of 4 . This quantity is shown in the inset of Fig. 3 as a function of the detector asymmetry. One finds that it approaches the value of 3. Thus an increase of the asymmetry considerably improves the efficiency of the SET although it does not reach the effectiveness of an ideal detector.

In addition, the effectiveness of measurement can be represented by the integrated signal-to-noise ratio $[15,5] s / n=\int_{-\infty}^{\infty}\left[\left|I_{s i g}(\omega)\right|^{2} / S(\omega)\right] d \omega / 2 \pi$. In our case the signal corresponds to the deviation of the detector current from its stationary value. Thus, $I_{\text {sig }}(\omega)=$ $\int_{0}^{\infty} \Delta I(t) \exp (i \omega t) d t$, where $\Delta I(t)=I(t)-I(t \rightarrow \infty)$. Using Eqs. (2), (3) and (5) one can evaluate the integrated signal-to-noise ratio as a function of $\Gamma_{R} / \Gamma_{L}$. The results of our calculations for $\epsilon=0, \Gamma_{L}=0.5 \Omega$ and $U=20 \Omega$ are shown in the inset of Fig. 3. As expected, the signal-to-noise ratio increases with $\Gamma_{R} / \Gamma_{L}$.

Although our calculations dealt with a strongly coupled detector, we anticipate that the asymmetric SET would be more effective than the symmetric one even for a weak coupling or for a metallic SET. Indeed the weight of the configurations (b) and (d) in Fig. 1 is always suppressed for $\Gamma_{R} \gg \Gamma_{L}$. As a result, the asymmetric SET would distort the measured system less than the symmetric one. In fact, this seems to be at variance with Refs. [6], which predict high effectiveness of the symmetric SET in the weak coupling regime. Yet recent analysis [5] that demonstrates low effectiveness of a symmetric SET in the weak coupling regime, supports our qualitative arguments. In addition, Refs. [4] also predict low effectiveness of the symmetric SET in the weakly responding regime.

In is interesting to compare qubit measurements using SET and PC detectors. The latter, considered as an ideal detector can be schematically represented by a tunneling barrier separating the left and the right reservoirs (instead of the two tunneling barriers in the SET, Fig. 1). The height of this barrier is modulated by the electrostatic field of the electron in the qubit. As a result, the current flowing through the point contact varies from $I_{1}$, when the electron occupies the level $E_{1}$ of the qubit, to $I_{2}$, if the electron occupies the level $E_{2}$ (Fig. 1). One finds that in the case of $E_{1}=E_{2}$ the qubit density matrix, $\sigma_{Q B}(t)$ is described by the following Bloch-type rate equations

$$
\begin{aligned}
& \dot{\sigma}_{11}=i \Omega\left(\sigma_{12}-\sigma_{21}\right), \\
& \dot{\sigma}_{12}=i \Omega\left(2 \sigma_{11}-1\right)-\left(\Gamma_{d} / 2\right) \sigma_{12},
\end{aligned}
$$

with the decoherence rate $\Gamma_{d}=\left(\sqrt{I_{1} / e}-\sqrt{I_{2} / e}\right)^{2}[16]$. This result supports the idea that, for the case of an ideal detector, the measurement-induced decoherence rate is directly related to the detector signal, or more precisely, to the ability of the detector to distinguish between different states of the qubit [13,17]. Indeed, the same expression for the decoherence rate can be obtained using a Bayesian formalism that shows the tendency of the qubit 
to evolve corresponding to the information acquired from an ideal measurement [4].

It is interesting that in the case of asymmetric SET detector and large $U$ the qubit density matrix is described by the same Eqs. (6) as in the case of PC (ideal) detector. This can be obtained by tracing over $n$ in Eqs. (2) and neglecting small terms of the order of $\Gamma_{L} / \Gamma_{R}, \Gamma_{L, R} / U$ and $\Omega / U$. As a result, one arrives to Eqs. (6), with the decoherence rate $\Gamma_{d}=\Gamma_{L}$. The latter corresponds to $I_{1}=I_{0} \rightarrow e \Gamma_{L}$ and $I_{2}=0$. Thus, $\Gamma_{d}$ is directly related to the detector signal as in the case of the PC detector [4]. Nevertheless the asymmetric SET does not become an ideal one, even in the large asymmetry limit, $\Gamma_{L} / \Gamma_{R} \ll 1$. Indeed, the peak-to-background ratio reaches the value 3 for the asymmetric SET (Fig. 3), whereas it becomes 4 for the PC detector $[4,14,18]$.

The above result might question the possible connection of decoherence with information gain. Indeed, it is assumed that in the case of a non-ideal detector (the SET) a part of the decoherence rate is generated by "pure environment" [4], in addition to that related to the measurement rate $[13,17]$. Yet, it follows from our analysis that the decoherence rate generated by the asymmetric SET is given by the same expression as that of the PC detector, i.e. it is related to the measurement only. Next, one could anticipate that the decoherence rate diminishes with the detector signal, since then only the "pure environment" would contribute to decoherence. This can be expected, for instance by increasing the chemical potential $\mu_{L}$ in Fig. 1, so that $\mu_{L} \gg E_{0}+U$. As a result the detector current becomes the same for the both states of the qubit: $I_{1}=I_{2}=I_{0} \simeq e \Gamma_{L}$. This case can be studied with the corresponding rate equations, similar to Eqs. (2). As in the previous case one finds that in the limit of $\Gamma_{L} \ll \Gamma_{R}$ and $U \gg \Gamma_{L, R}$ the qubit density matrix is given by Eqs. (6), but with $\Gamma_{d}=2 \Gamma_{L}$.

Such an increase of $\Gamma_{d}$ in the no-measurement regime can be understood if the decoherence generated by the SET is associated with the detector shot-noise, rather than the information flow. Indeed, the shot-noise increases by a factor of two with respect to the measurement case. The reason is that in the measurement regime the detector is blocked whenever the electron occupies the state $E_{2}$ of the qubit, Fig. 1. In any case, irrespective of any possible interpretation, our results demonstrate that the reduction of information acquired by a measurement, may not lead to a decrease of the decoherence rate, even for a nearly ideal detector.

In summary, we have found that the effectiveness of the SET detector can be considerably increased by making it asymmetric, where the tunneling to the collector is much faster than the tunneling to the emitter reservoir. In this case the effectiveness of the detector becomes more like that of the PC detector. Yet the decoherence rate behaves differently when no signal can be extracted from the measurement. In particular, the decoherence rate in- creases by a factor of two in the case of the SET, whereas it vanishes for the PC detector. This questions the role of information gain in the decoherence mechanism. We believe that this result should be taken into account in any consideration of how to diminish the decoherence of a single quantum system interacting with a detector.

One of the authors (S.G.) acknowledges useful discussions with D. Mozyrsky. This work was supported by the Department of Energy (DOE) under Contract No. W7405-ENG-36, by the National Security Agency (NSA), and by the Advanced Research and Development Activity (ARDA) contract under Army Research Office (ARO) \# 707003 .

[1] M.H. Devoret and R.J. Schoelkopf, Nature 406, 1039 (2000).

[2] Yu. Makhlin, G. Schön, and A. Shnirman, Rev. Mod. Phys. 73, 357 (2001).

[3] E. Buks, R. Shuster, M. Heiblum, D. Mahalu and V. Umansky, Nature 391, 871 (1998).

[4] A.N. Korotkov, Phys. Rev. B63, 085312 (2001); ibid B63, 115403 (2001).

[5] D. Mozyrsky, I. Martin, and M.B. Hastings, Phys. Rev. Lett. 92, 018303 (2004).

[6] D.V. Averin, Fortschr. Phys. 48, 1055 (2000); A.A. Clerk and A.D. Stone, Phys. Rev. B69, 245303 (2004).

[7] In fact, our treatment remains essentially the same for many conducting levels contributing to transport, providing that their widths do not overlap (c.f. with [2]). Otherwise, the treatment should be modified.

[8] Although the state $1 \mathrm{~d}$ is not directly accessible due to transitions from the left reservoir, it can reached in the course of the Rabi oscillations of the qubit if $\Omega$ and $U$ are comparable.

[9] R.H. Dicke, Am. J. Phys. 49, 925(1981); A. Elitzur and L. Vaidman, Found. Phys. 23, 987 (1993); P. Kwiat, H. Weinfurter, T. Herzog, A. Zeilinger, M.A. Kasevich, Phys. Rev. Lett. 74, 4763 (1995).

[10] S.A. Gurvitz and Ya.S. Prager, Phys. Rev. B53, 15932 (1996); S.A. Gurvitz, Phys. Rev. B57, 6602 (1998).

[11] S.A. Gurvitz, Phys. Lett. A311, 292 (2003).

[12] D. Mozyrsky, L. Fedichkin, S.A. Gurvitz and G.P. Berman, Phys. Rev. B66, 161313(R) (2002).

[13] D.V. Averin, cond-mat/0301524.

[14] H.S. Goan and G.J. Milburn, Phys. Rev. B64, 235307 (2001).

[15] V.B. Braginsky and F.Ya. Khalili, Quantum Measurement, Cambridge University Press (1992).

[16] S.A. Gurvitz, Phys. Rev. B56, 15215 (1997).

[17] A.A. Clerk, S.M. Girvin and A.D. Stone, Phys. Rev. B67, 165324 (2003).

[18] It was argued, however, in Refs. [4] that in the case of a weak response the asymmetric SET becomes an ideal detector for $\Gamma_{R} / \Gamma_{L} \gg 1$. 